\title{
Gold-style and query learning under various constraints on the target class
}

\author{
Sanjay Jain, ${ }^{1 \star}$ Steffen Lange, ${ }^{2}$ and Sandra Zilles ${ }^{3}$ \\ 1 School of Computing, National University of Singapore, Singapore 117543, \\ e-mail: sanjay@comp.nus.edu.sg \\ 2 Fachhochschule Darmstadt, FB Informatik, Haardtring 100, 64295 Darmstadt, \\ Germany, e-mail: s.lange@fbi.fh-darmstadt.de \\ 3 DFKI GmbH, Erwin-Schrödinger-Straße, 67663 Kaiserslautern, Germany, \\ e-mail: sandra.zilles@dfki.de
}

\begin{abstract}
In language learning, strong relationships between Gold-style models and query models have recently been observed: in some quite general setting Gold-style learners can be replaced by query learners and vice versa, without loss of learning capabilities. These 'equalities' hold in the context of learning indexable classes of recursive languages.

Former studies on Gold-style learning of such indexable classes have shown that, in many settings, the enumerability of the target class and the recursiveness of its languages are crucial for learnability. Moreover, studying query learning, non-indexable classes have been mainly neglected up to now. So it is conceivable that the recently observed relations between Gold-style and query learning are not due to common structures in the learning processes in both models, but rather to the enumerability of the target classes or the recursiveness of their languages.

In this paper, the analysis is lifted onto the context of learning arbitrary classes of r.e. languages. Still, strong relationships between the approaches of Gold-style and query learning are proven, but there are significant changes to the former results. Though in many cases learners of one type can still be replaced by learners of the other type, in general this does not remain valid vice versa. All results hold even for learning classes of recursive languages, which indicates that the recursiveness of the languages is not crucial for the former 'equality' results. Thus we analyse how constraints on the algorithmic structure of the target class affect the relations between two approaches to language learning.
\end{abstract}

\section{Introduction}

In order to model different aspects of human learning and machine learning, different abstract approaches have to be considered. Each model analysed within the scope of learning theory addresses only special facets of learning. For example, in Gold's [9] model of identification in the limit learning is interpreted as a limiting process of generating and improving hypotheses about a target concept. These hypotheses are built upon instances of the target concept offered to

\footnotetext{
* Sanjay Jain was supported in part by NUS grant number R252-000-127-112.
} 
the learner. In the limit, the output of the learner is supposed to stabilize on a correct guess, but during the learning process one never knows whether or not the current hypothesis is already correct. The potential of changing its mind is a crucial quality of the learner.

In contrast to that, Angluin's [3,4] model of query learning is concerned with learning as a finite process in which a learner and a teacher interact. The learner asks questions of a specified type about the target concept and the teacher answers these reliably. After finitely many steps the learner is required to return a single hypothesis, which then correctly describes the target concept. Here the crucial characteristics of the learner are its access to special information on the target concept and its confinements in terms of mind changes. Since a query learner identifies the target concept with just a single hypothesis, we allude to this scheme as one-shot learning. ${ }^{4}$

Recently, the combination of these two approaches [11,12] as well as the common features of learners in either model $[14,15]$ have gained interest in the learning theory community. $[14,15]$ contributes a systematic analysis of common features of both approaches, thereby focussing on the identification of formal languages, ranging over indexable classes of recursive languages, as target concepts, see $[2,13,19]$. Characterising different types of Gold-style language learning in terms of query learning has pointed out correspondences between the two models. In particular, $[14,15]$ demonstrate how learners identifying languages in the limit can be replaced by one-shot learners without loss of learning power-and vice versa. That means, under certain circumstances the capabilities of limit learners are equal to those of one-shot learners using queries. An important parameter in this context is the range of possible hypothesis spaces/query spaces used during the learning process. Despite the fundamental differences in the definitions of the two learning paradigms, there are strong relations - at least in the case of learning indexable families of recursive languages.

The latter restriction had initially been made, since many natural language classes are indexable. Former studies [19] on Gold-style learning of indexable classes of languages have shown that, in many settings, the enumerability of the target class may be the crucial reason for positive learnability results. Moreover, when studying query learning, non-indexable classes have been mainly neglected up to now. So it is conceivable that the strong relationships between Gold-style and query learning observed in $[14,15]$ are not caused by common structures in the learning processes in both models, but rather by the enumerability of the target classes or maybe at least by the recursiveness of the target languages themselves. In order to determine the actual cause for the relationships observed before, we now lift the analysis thereof onto more complex classes of languages.

Therefore the current paper concerns the relationships of Gold-style learning and query learning for the case that arbitrary classes of r.e. languages form the target. This is additionally based on the following observation: when trying to learn a class of recursive languages, a certain type of learner may sometimes be

\footnotetext{
${ }^{4}$ Most studies on query learning mainly deal with the efficiency of query learners, whereas, in what follows, we are only interested in qualitative learnability results.
} 
successful only in case the learner uses a hypothesis space comprising more than the languages to be learned - such as for instance a hypothesis space given by an r.e. indexing of r.e. languages. Then a natural question might be whether it is possible to learn not only the initial target class, but additionally the languages represented by further queries a learner asks or further hypotheses a learner states during learning the initial target languages. This again leads to the problem of learning r.e. languages. Literature, see e.g. [7], knows more examples of lifting results on learning recursive languages, as in [2], to learning r.e. languages.

From now on assume that arbitrary classes of r.e. languages form the target classes. Below we prove that in almost all cases, where equivalences between two learning models $A$ and $B$ had been witnessed for learning indexable classes of recursive languages, learners of type $A$ can be replaced by learners of type $B$ without loss of learning power-but no longer vice versa. So, although most of the equivalences between Gold-style models and query models no longer hold, at least some of the inclusions hold, thereby forming a hierarchy of inference types. This shows that huge parts of the relationships shown for learning indexable classes of recursive languages are maintained; the cause must be common structures of learning processes in Gold-style and query learning! An important parameter in the final hierarchy is again the underlying hypothesis space/query space.

Interestingly, all separations of inference types in the final hierarchy can be witnessed even by (non-indexable) classes of recursive languages. This raises the question whether the main reason for the equivalence results in $[14,15]$ is the fact that the classes considered are enumerable and not that the languages themselves are recursive. So we analysed whether the results in $[14,15]$ can be lifted to the case of learning enumerable classes of r.e. languages. The relationships observed are somewhat dismal: several of the equivalence results do not hold for learning enumerable classes of r.e. languages, but at least one of them does. That means that in most but not in all cases, the main reason for the equivalence results in $[14,15]$ lies not only in the enumerability of the target classes.

\section{Preliminaries}

Familiarity with standard recursion theoretic notions is assumed, see $[17,10]$. From now on, a fixed finite alphabet $\Sigma$ with $\{a, b\} \subseteq \Sigma$ is given. A word is any element from $\Sigma^{*}$ and a language any subset of $\Sigma^{*}$. The complement of a language $L$, denoted $\bar{L}$, is the set $\Sigma^{*} \backslash L$. Any total function $t: \mathbb{N} \rightarrow \Sigma^{*}$ with $\{t(i) \mid i \in \mathbb{N}\}=L$ is called a text for $L$. A text $t$ is often identified with an infinite sequence $(t(i))_{i \in \mathbb{N}}$. Then, given $n \in \mathbb{N}, t_{n}$ is the initial segment $(t(0), \ldots, t(n))$ and content $\left(t_{n}\right)$ denotes the set $\{t(0), \ldots, t(n)\}$.

In the sequel, $\varphi$ is a Gödel numbering of all partial recursive functions and $K=\left\{i \in \mathbb{N} \mid \varphi_{i}(i)\right.$ is defined $\}$. The language family $\left(W_{i}\right)_{i \in \mathbb{N}}$ is given by $W_{i}=$ $\left\{w_{j} \mid \varphi_{i}(j)\right.$ is defined $\}$ for all $i \in \mathbb{N}$, where $\left(w_{j}\right)_{j \in \mathbb{N}}$ is a repetition-free effective enumeration of $\Sigma^{*}$. Then $W_{i, s}, s \in \mathbb{N}$, is the set of all words $w_{j}$, such that $j<s$ and $\varphi_{i}(j)$ terminates within $s$ steps. Given $A \subseteq \mathbb{N}$, an $A$-recursive function is a function recursive using an oracle for the set $A$. 
A family $\left(A_{i}\right)_{i \in \mathbb{N}}$ of languages is uniformly recursive (uniformly r.e.) if there is a recursive (partial recursive) function $f$ such that $A_{i}=\left\{w \in \Sigma^{*} \mid f(i, w)=1\right\}$ for all $i \in \mathbb{N}$. For uniformly recursive families membership is uniformly decidable. A family $\left(A_{i}\right)_{i \in \mathbb{N}}$ is uniformly $K$-r.e., if there is a recursive function $g$ such that $A_{i}=\left\{w \in \Sigma^{*} \mid g(i, w, n)=1\right.$ for all but finitely many $\left.n\right\}$ for all $i \in \mathbb{N}$. A class $\mathcal{C}$ of recursive languages over $\Sigma^{*}$ is called an indexable class of recursive languages (or indexable class for short), if there is a uniformly recursive family $\left(L_{i}\right)_{i \in \mathbb{N}}$ of all and only the languages in $\mathcal{C}$.

\subsection{Gold-style language learning}

Let $\mathcal{C}$ be a class of r.e. languages, $\mathcal{H}=\left(A_{i}\right)_{i \in \mathbb{N}}$ a language family (a hypothesis space). An inductive inference machine (IIM for short) $M$ is an algorithmic device that reads longer and longer initial segments $\sigma$ of a text and outputs numbers $M(\sigma)$. Returning $i, M$ is construed to hypothesize the language $A_{i}$.

The following definition of learning in the limit is based on [9]. Given a text $t$ for $L \in \mathcal{C}, M$ identifies $L$ from $t$ with respect to $\mathcal{H}=\left(A_{i}\right)_{i \in \mathbb{N}}$ in the limit, if the sequence of hypotheses output by $M$, when fed $t$, stabilizes on a number $i$ (i.e., past some point $M$ always outputs the hypothesis $i$ ) with $A_{i}=L$. $M$ identifies $\mathcal{C}$ in the limit from text with respect to $\mathcal{H}$, if it identifies every $L^{\prime} \in \mathcal{C}$ from every text for $L^{\prime}$. In what follows, we focus our studies on uniformly r.e. families as hypothesis spaces. $\operatorname{Lim} T x t_{\text {r.e. }}$ denotes the collection of all classes $\mathcal{C}^{\prime}$ for which there is a uniformly r.e. hypothesis space $\mathcal{H}$ and an IIM $M^{\prime}$ identifying $\mathcal{C}^{\prime}$ in the limit from text with respect to $\mathcal{H}$. A quite natural and often studied modification of LimTxt r.e. is defined by the model of conservative inference, see $[2,13]$ for this concept in the context of learning recursive languages. $M$ is a conservative IIM for $\mathcal{C}$ with respect to $\mathcal{H}=\left(A_{i}\right)_{i \in \mathbb{N}}$, if $M$ performs only justified mind changes, i.e., if $M$, on some text $t$ for some $L \in \mathcal{C}$, outputs hypotheses $i$ and later $j$, then $M$ must have seen some element $w \notin A_{i}$ before returning $j$. The collection of all classes identifiable from text by a conservative IIM with respect to some uniformly r.e. hypothesis space is denoted by ConsvTxt $t_{\text {.e. }}$. Note that ConsvTxt r.e. $\subset \operatorname{LimTxt}_{\text {r.e. }}$, as witnessed by the indexable class used in [19] to separate LimTxt-learnable indexable classes from ConsvTxt-learnable indexable classes. Another often studied version of Gold-style language learning is behaviourally correct learning [6]: If $\mathcal{C}$ is a class of r.e. languages, $\mathcal{H}=\left(A_{i}\right)_{i \in \mathbb{N}}$ any hypothesis space, $M$ an IIM, then $M$ is a behaviourally correct learner for $\mathcal{C}$ from text with respect to $\mathcal{H}$, if for each $L \in \mathcal{C}$ and each text $t$ for $L$, for all but finitely many $n, A_{M\left(t_{n}\right)}=L$ is fulfilled. Here $M$ may alternate different correct hypotheses arbitrarily often instead of converging to a single hypothesis. Defining the notion $B c T x t_{\text {r.e. }}$ as usual yields $B c T x t_{\text {r.e. }} \supset \operatorname{Lim} T x t_{\text {r.e. }}$ [6].

Since we analyse learning from text, we assume in the sequel that all target languages are non-empty. One main aspect of human learning is modelled in the approach of learning in the limit: the ability to change one's mind. Thus learning is a process in which the learner may change its hypothesis arbitrarily often until reaching its final correct guess. In particular, it is in general impossible to find out when the final hypothesis has been reached, i.e., when a success in learning 
has eventuated. The main concern of our analysis will be comparisons of such inference types to query learning models resulting in a hierarchy reflecting the capabilities of the corresponding learners.

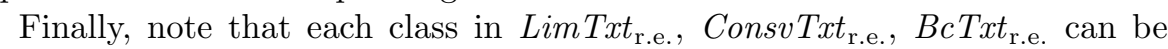
learned using the hypothesis space $\left(W_{i}\right)_{i \in \mathbb{N}}$. We will use this property in our proofs below. These notions of learning are closely related to the notion of stabilizing sequences [8]. If $\mathcal{H}=\left(A_{i}\right)_{i \in \mathbb{N}}$ is a hypothesis space, $M$ an IIM, and $L$ a language, then any finite text segment $\sigma$ of $L$ is called a $\operatorname{Lim} T x t$-stabilizing sequence (a BcTxt-stabilizing sequence) for $M, L$, and $\mathcal{H}$, if $M(\sigma)=M\left(\sigma \sigma^{\prime}\right)$ $\left(A_{M(\sigma)}=A_{M\left(\sigma \sigma^{\prime}\right)}\right)$ for all finite text segments $\sigma^{\prime}$ of $L$. If $L$ is $\operatorname{Lim} T x t$-learned by $M(B c T x t$-learned by $M)$ respecting $\mathcal{H}$, then there exists a LimTxt-stabilizing sequence (a BcTxt-stabilizing sequence) for $M, L$, and $\mathcal{H}$.

\subsection{Language learning via queries}

In the query learning model, a learner has access to a teacher that truthfully answers queries of a specified kind. A query learner $M$ is an algorithmic device that, depending on the reply on the previous queries, either computes a new query or returns a hypothesis and halts [3]. Its queries and hypotheses are coded as natural numbers; both will be interpreted with respect to an underlying hypothesis space. We adapt Angluin's original definition here for learning r.e. languages as follows: when learning a class $\mathcal{C}$ of r.e. languages, any family $\left(A_{i}\right)_{i \in \mathbb{N}}$ of languages may form a hypothesis space.

More formally, let $\mathcal{C}$ be a class of r.e. languages, let $L \in \mathcal{C}$, let $\mathcal{H}=\left(A_{i}\right)_{i \in \mathbb{N}}$ be a hypothesis space, let $M$ be a query learner. $M$ learns $L$ with respect to $\mathcal{H}$ using some type of queries if it eventually halts and its only hypothesis, say $i$, represents $L$, i.e., $A_{i}=L$. So $M$ returns its unique and correct guess $i$ after finitely many queries. Moreover, $M$ learns $\mathcal{C}$ with respect to $\mathcal{H}$ using some type of queries, if it learns every $L^{\prime} \in \mathcal{C}$ with respect to $\mathcal{H}$ using queries of the specified type. If $L$ is a target language, a query learner $M$ may ask:

Restricted superset queries. The input is an index of a language $L^{\prime} \in \mathcal{C}$. The answer is 'yes' or 'no', depending on whether or not $L$ ' is a superset of $L$.

Restricted disjointness queries. The input is an index of a language $L^{\prime} \in \mathcal{C}$. The answer is 'yes' or 'no', depending on whether or not $L^{\prime}$ and $L$ are disjoint.

The term 'restricted' is used to distinguish these inference types from learning with superset (disjointness) queries, where, with each negative reply to a query $j$ the learner is provided a counterexample, i.e., a word in $L \backslash A_{j}$ (in $L \cap A_{j}$ ).

$S u p Q_{\text {r.e. }}$ and $D i s Q_{\text {r.e. }}$ denote the collections of all classes $\mathcal{C}^{\prime}$ for which there is a uniformly r.e. hypothesis space $\mathcal{H}$ and a query learner $M^{\prime}$ learning $\mathcal{C}^{\prime}$ with respect to $\mathcal{H}$ using restricted superset and restricted disjointness queries, respectively. In the sequel we will omit the term 'restricted' for convenience and will again without loss of generality assume that $\operatorname{Sup} Q_{\text {r.e.- }}$-learners and $D i s Q_{\text {r.e.' }}$ learners always use the hypothesis space $\mathcal{H}=\left(W_{i}\right)_{i \in \mathbb{N}}$. In the literature, see Angluin $[3,4]$, more types of queries, such as (restricted) subset queries, membership 
queries, and equivalence queries have been analysed, but in what follows we concentrate on the two types explained above. Obviously, superset and disjointness queries are in general not decidable, i.e., the teacher may be non-computable.

Note that, in contrast to the models of Gold-style language learning introduced above, learning via queries focusses the aspect of one-shot learning, i.e., it is concerned with scenarios in which learning eventuates without mind changes.

\section{Learning indexable classes of recursive languages}

Numerous studies on language learning restrict their focus on indexable classes, since, first, these include many natural classes of languages, and second, many conceptions can be simplified in this context. In particular, uniformly recursive families may be considered as hypothesis spaces in the approaches of both Goldstyle and query learning (indicated by a subscript rec instead of r.e.). In this section, all results referred to hold for indexable classes only. Recent studies $[14,15]$ have shown astonishing relations between the two approaches witnessed by equivalences of pairs of inference types, such as $\operatorname{Sup} Q_{\text {rec }}=D i s Q_{\text {rec }}=\operatorname{ConsvTxt}_{\text {r.e. }}$ $\left(=\right.$ ConsvTxt $_{\text {rec }}$, see a result by Jain in [16] $)$ and $D i s Q_{\text {r.e. }}=\operatorname{LimTxt}_{\text {r.e. }}$. In these equalities, all inference types are considered restricted to indexable classes.

Concerning characterisations of $\operatorname{Sup} Q_{\text {r.e. }}$ and $B c T x t_{\text {rec }}$ by similar means, oracle-IIMs as well as more general hypothesis spaces have been useful. Firstly, an oracle-IIM is an IIM which is recursive relative to an arbitrary oracle, i.e. its computation depends on according to which oracle it currently accesses, see e.g. [18]. For instance, using a $K$-oracle, such an IIM $M$ becomes a $K$-recursive IIM $M^{K}$. Thus, e.g., ConsvTxt $t_{\text {r.e. }}[K]$ denotes the collection of classes ConsvTxt $t_{\text {r.e.- }}$ learnable with the help of a $K$-oracle. Restricting such inference types to index-

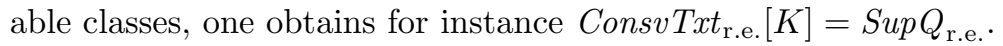

Secondly, in order to characterise $B c T x t_{\text {r.e. }}$, uniformly $K$-r.e. hypothesis spaces have been introduced for query learning, indicated by a subscript $K$-r.e. as in $\operatorname{Sup} Q_{K \text {-r.e. }}$. This has lead to the result $\operatorname{Sup} Q_{K \text {-r.e. }}=\operatorname{Dis} Q_{K \text {-r.e. }}=B c T x t_{\text {r.e. }}$.

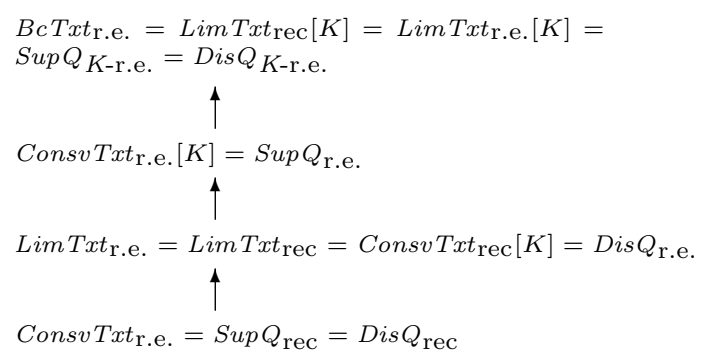

Figure 1. This graph illustrates the relations between different inference types restricted to indexable classes as studied in [14, 15]. Arrows indicate proper inclusions of inference types.

\section{Learning classes of r.e. languages}

In the sequel, a hierarchy as in Figure 1 is established for arbitrary classes of r.e. languages. A section on query learning with uniformly r.e. hypothesis spaces is followed by a section dealing with uniformly $K$-r.e. hypothesis spaces. Note that indexable hypothesis spaces in general are obsolete here. 


\subsection{Results for uniformly r.e. hypothesis spaces}

Our first comparison already yields a change to the former hierarchy: when learning arbitrary classes of r.e. languages, ConsvTxt $t_{\text {r.e. }}$-learners can in general no longer be replaced by $D i s Q_{\text {r.e. }}$-learners.

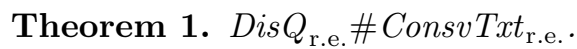

Proof. Dis $Q_{\text {r.e. }} \backslash$ ConsvTxt $t_{\text {r.e. }} \neq \emptyset$ follows from Figure 1. For ConsvTxt $t_{\text {r.e. }} \backslash$ $D i s Q_{\text {r.e. }} \neq \emptyset$, consider the following class $\mathcal{C}$.

Let $L_{i}=\left\{a^{i} b^{x} \mid x \in \mathbb{N}\right\}$. Let $L_{i}^{S}=\left\{a^{i} b^{x} \mid x \in S\right\}$ for any set $S \subseteq \mathbb{N}$.

Let $\mathcal{C}_{1}=\left\{L_{i}^{S} \mid i \in \mathbb{N}, \operatorname{card}(S)<\infty, \exists e[\operatorname{card}(S \cap\{x \mid x \leq 2 e\})>e+1]\right\}$.

Below, we will define a recursive function $f$ such that, for all $i$, the following two properties hold:

(a) $W_{f(i)} \subseteq L_{i}$ and $W_{f(i)}$ is recursive (although an index for the characteristic function of $W_{f(i)}$ in general cannot be obtained from $i$ );

(b) For all $e, \operatorname{card}\left(W_{f(i)} \cap\left\{a^{i} b^{x} \mid x \leq 2 e\right\}\right) \leq e+1$.

Let $\mathcal{C}_{2}=\left\{W_{f(i)} \mid i \in \mathbb{N}\right\}$. Let $\mathcal{C}=\mathcal{C}_{1} \cup \mathcal{C}_{2}$. Thus, $\mathcal{C}$ is uniformly r.e. and consists only of recursive languages (however, $\mathcal{C}$ is not an indexed family).

$\mathcal{C} \in$ ConsvTxt r.e. is witnessed by an IIM $M$ which, on target $L$, first acquires an $i$ with $L \subseteq L_{i}$. $M$ outputs $f(i)$, until an $e$ with $\operatorname{card}\left(\left\{x \mid a^{i} b^{x} \in L, x \leq 2 e\right\}\right)>$ $e+1$ is found. In the latter case $M$ runs the learning procedure for finite sets.

Let $\left(M_{i}\right)_{i \in \mathbb{N}}$ be an enumeration of all Dis $Q_{\text {r.e. }}$-learners. We now define $f$, such that (a) and (b) above are fulfilled and for each $i, M_{i}$ either does not $D i s Q_{\text {r.e. }}$-identify $W_{f(i)}$, or it does not $D i s Q_{\text {r.e. }}$-identify $\mathcal{C}_{1}$.

For any $i, s \in \mathbb{N}$, let $W_{f(i)}^{s}$ denote the subset of $W_{f(i)}$ enumerated before stage $s$. Let $W_{f(i)}^{0}=\left\{a^{i}\right\}$, i.e., the word $a^{i}$ is enumerated in $W_{f(i)}$ before stage 0 . Go to stage 0 . In general, stage $s$ reads as follows.

- Step 1: Run $M_{i}$ for $s$ steps, where each disjointness query $j$ (representing $W_{j}$ ) of $M_{i}$ is answered 'yes', if $W_{j, s} \cap W_{f(i)}^{s}=\emptyset$; 'no' otherwise.

- Step 2: If $M_{i}$ does not output a hypothesis within $s$ steps, go to stage $s+1$. Else dovetail steps 2.1 and 2.2 until one of them succeeds. If 2.1 succeeds before 2.2, then go to stage $s+1$, else if 2.2 succeeds, then go to step 3 .

(2.1) Find a query $j$ from step 1 which was answered 'yes', but $W_{j} \cap W_{f(i)}^{s} \neq \emptyset$.

(2.2) Find a query $j$ from step 1 which was answered 'yes', and $a^{i} b^{y} \in W_{j}$ for some $y>2 s$.

- Step 3: Let $j, y$ be as found in step 2.2. Enumerate $a^{i} b^{y}$ in $W_{f(i)}$ and go to stage $s+1$ (otherwise stage $s$ never ends).

Fix $i$. By construction, $W_{f(i)}$ fulfils the conditions (a) (as either $W_{f(i)}$ is finite, or $a^{i} b^{s} \in W_{f(i)}$, iff it is enumerated in $W_{f(i)}$ before stage $s$ ) and (b) (as at most $s+1$ elements are enumerated before stage $s$, and every element enumerated at or after stage $s$ is of form $a^{i} b^{y}$ for some $y>2 s$ ). We consider two cases.

Case 1: Stage s starts but does not finish. In this case clearly $W_{f(i)}$ is finite. Now, since step 2.1 did not succeed, all questions of $M_{i}$ in step 1 above for the input being $W_{f(i)}=W_{f(i)}^{s}$, are answered correctly at stage $s$, and $M_{i}$ outputs 
a hypothesis on $W_{f(i)}$. Furthermore, all questions $j$ of $M_{i}$ on $W_{f(i)}$ which are answered 'yes', have the property that $W_{j} \cap L_{i}$ is finite (since step 2.2 did not succeed). Thus, there exists a finite set $S$ with $L_{i}^{S} \in \mathcal{C}_{1}$ such that $M_{i}$ behaves the same way on $L_{i}^{S}$ as it does on $W_{f(i)}$. To see this, let $S=\left\{x \mid a^{i} b^{x} \in W_{f(i)}\right\} \cup\{z \mid$ $e \leq z \leq 2 e\}$, where $e=1+\max \left(\left\{y \mid a^{i} b^{y} \in W_{j}\right.\right.$ for some query $j$ asked by $M_{i}$ on input $W_{f(i)}$, and answered 'yes' $\}$ ) (* note that for each question $j$ asked by $M_{i}$ on input $W_{f(i)}$ and answered 'yes', $W_{j} \cap L_{i}$ is finite $\left.{ }^{*}\right)$. Now, $M_{i}$ can $D i s Q_{\text {r.e. }}$-identify at most one of $W_{f(i)}$ and $L_{i}^{S}$, both of which are in $\mathcal{C}$.

Case 2: Every stage $s$ ends. Consider the following subcases:

Case 2.1: $M_{i}$ on $W_{f(i)}$ asks infinitely many questions or never outputs a hypothesis. In this case clearly, $M_{i}$ does not $D i s Q_{\text {r.e. }}$-identify $W_{f(i)} \in \mathcal{C}$.

Case 2.2: Not case 2.1. In this case let stage $s$ be large enough so that, if $j$ is a question asked by $M_{i}$ on $W_{f(i)}$ (when all the questions are answered correctly), and $W_{j} \cap W_{f(i)} \neq \emptyset$, then $W_{j, s} \cap W_{f(i)}^{s} \neq \emptyset$. Note that then beyond stage $s$ all questions of $M_{i}$ are answered correctly in step 1. Now step 2.1 and 2.2 cannot succeed. Thus the only way infinitely many stages can exist is by $M_{i}$ not returning any hypothesis. A contradiction.

From the above cases it follows that $M_{i}$ does not $D i s Q_{\text {r.e. }}$-identify $\mathcal{C}$.

Thus we obtain Dis $Q_{\text {r.e. }} \#$ ConsvTxt $t_{\text {r.e. }}$.

This incoherency holds since presently $D i s Q_{\text {r.e. }}$ no longer equals $\operatorname{Lim} T x t_{\text {r.e. }}$. However, an inclusion as in Thm. 2 still indicates a relation between Gold-style and query learning, albeit weaker than when restricted to indexable classes.

Theorem 2. $D i s Q_{\text {r.e. }} \subset \operatorname{Lim} T x t_{\text {r.e. }}$.

Proof. For the inclusion the congruent proof for indexable classes in [14] can be adopted. The inequality follows from Thm. 1 and ConsvTxt $t_{\text {r.e. }} \subseteq \operatorname{Lim}_{\mathrm{T} x t_{\text {r.e. }}}$.

The relationship between $\operatorname{Lim} T x t_{\text {r.e. }}$ and $\operatorname{Sup} Q_{\text {r.e. }}$ remains unchanged from the former hierarchy, as Thm. 3 shows.

Theorem 3. $\operatorname{Lim} T x t_{\text {r.e. }} \subset \operatorname{Sup} Q_{\text {r.e. }}$.

Proof. The proof of $\operatorname{Lim} T x t_{\text {r.e. }} \subseteq \operatorname{Sup} Q_{\text {r.e. }}$ is omitted. The underlying idea is similar to that in the proof of $\operatorname{Lim} T x t_{\text {r.e. }} \subseteq \operatorname{Dis} Q_{\text {r.e. }}$ in [14]. $\operatorname{Sup} Q_{\text {r.e. }} \backslash \operatorname{Lim} T x t_{\text {r.e. }} \neq \emptyset$ is even witnessed by a uniformly recursive family of languages, see [14].

Interestingly, the characterisation $\operatorname{Sup} Q_{\text {r.e. }}=\operatorname{Consv} T x t_{\text {r.e. }}[K]$ persists when learning classes of r.e. languages. Here the proof for indexable classes [15] applies.

Theorem 4. $\operatorname{Sup} Q_{\text {r.e. }}=\operatorname{Consv} T x t_{\text {r.e. }}[K]$.

Though $\operatorname{Sup} Q_{\text {r.e. }} \subset \operatorname{Lim} \operatorname{Txt}_{\mathrm{r} . \mathrm{e}}[K]$ persists $($ Thm. 5 ), the relation between $S u p Q_{\text {r.e. }}$ and $B c T x t_{\text {r.e. }}$ changes significantly for arbitrary classes of r.e. languages, see Thm. 7. The reason is that $\operatorname{Lim} T x t_{\text {r.e. }}[K]$ no longer equals $B c T x t_{\text {r.e. }}$ (Thm. 6).

Theorem 5. $\operatorname{Sup} Q_{\text {r.e. }} \subset \operatorname{Lim} T x t_{\text {r.e. }}[K]$.

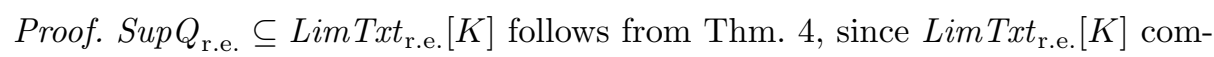
prises ConsvTxt r.e. $[K]$. As BcTxt r.e. $\backslash$ ConsvTxt r.e. $[K] \neq \emptyset[15]$, Thm. 4 yields

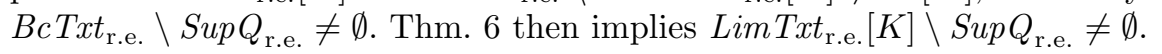


Theorem 6. $B c T x t_{\text {r.e. }} \subset \operatorname{Lim} T x t_{\text {r.e. }}[K]$.

Proof. To show $B c T x t_{\text {r.e. }} \subseteq \operatorname{Lim} T x t_{\text {r.e. }}[K]$ suppose $\mathcal{C}$ is a class of r.e. languages in $B c T x t_{\text {r.e. }}$. Let $M$ be an IIM identifying $\mathcal{C}$ behaviourally correctly in $\left(W_{i}\right)_{i \in \mathbb{N}}$.

The following oracle-IIM $M^{\prime}$ LimTxt-identifies $\mathcal{C}$ with respect to $\left(W_{i}\right)_{i \in \mathbb{N}}$ using an oracle for $K$. Given a text segment $t_{n}$ of length $n+1, M^{\prime}$ first computes $M\left(t_{n}\right)$. If $n=0$, then $M^{\prime}\left(t_{n}\right)=M\left(t_{n}\right)$. If $n>0$, then $M^{\prime}$ uses the $K$-oracle to determine whether or not there is a word $w_{x}$ for some $x \leq n$, such that $w_{x} \in W_{M\left(t_{n}\right)} \Delta W_{M^{\prime}\left(t_{n-1}\right)}$. If no such word exists, then $M^{\prime}\left(t_{n}\right)=M^{\prime}\left(t_{n-1}\right)$. Else $M^{\prime}\left(t_{n}\right)=M\left(t_{n}\right)$. It is not hard to prove that $M^{\prime}$ learns all languages in $\mathcal{C}$ in the limit respecting $\left(W_{i}\right)_{i \in \mathbb{N}}$. Thus $B c T x t_{\text {r.e. }} \subseteq \operatorname{Lim} T x t_{\text {r.e. }}[K]$.

$\operatorname{LimTxt}_{\text {r.e. }}[K] \backslash B c T x t_{\text {r.e. }} \neq \emptyset$ is witnessed by the class $\mathcal{C}_{R}=\left\{L_{f} \mid f\right.$ is a recursive function $\}$, where for each partial recursive function $f$ we define $L_{f}=$ $\left\{a^{x} b^{f(x)} \mid x \in \mathbb{N}\right\} .\left({ }^{*} \mathcal{C}_{R}\right.$ consists only of recursive languages. $\left.{ }^{*}\right)$ If $\mathcal{C}_{R}$ was $B c T x$-learnable, then the class of all recursive functions would be $B c$-learnable as defined in [5]. The latter contradicts a result in [5]. On the other hand, $\mathcal{C}_{R}$ is $\operatorname{Sup} Q_{\text {r.e. }}$-learnable: if $L \in \mathcal{C}_{R}$ is the target language, a learner can find the least $i$ with $L_{\varphi_{i}} \supseteq L$. Then $L_{\varphi_{i}}$ must equal $L$. By Thm. 4 , then $\mathcal{C}_{R} \in \operatorname{Consv} T x t_{\text {r.e. }}[K] \subseteq$ $\operatorname{LimTxt}_{\text {r.e. }}[K] .^{5}$ This establishes $B c T x t_{\text {r.e. }} \subset \operatorname{Lim} T x t_{\text {r.e. }}[K]$.

Theorem 7. BcTxt r.e. \# Sup $Q_{\text {r.e. }}$.

Proof. For $B c T x t_{\text {r.e. }} \backslash S u p Q_{\text {r.e. }} \neq \emptyset$ see [15]. The class $\mathcal{C}_{R}$ used to prove Thm. 6 witnesses $\operatorname{Sup} Q_{\text {r.e. }} \backslash B c T x t_{\text {r.e. }} \neq \emptyset$.

\subsection{Results for uniformly $K$-r.e. hypothesis spaces}

Finally, we consider $K$-r.e. hypothesis spaces for query learning as in [14,15]. A family $\left(A_{i}\right)_{i \in \mathbb{N}}$ is uniformly $K$-r.e., if there is a recursive function $g$ with $A_{i}=\left\{w \in \Sigma^{*} \mid g(i, w, n)=1\right.$ for all but finitely many $\left.n\right\}$ for all $i \in \mathbb{N}$. As it turns out, all the equality results from former studies, as illustrated in Figure 1, now turn into proper inclusions. So, though there are strong relations between the corresponding inference types, these are not as strong as in the context of learning indexable classes. Thms. 8 and 9 state this formally.

Theorem 8. $\operatorname{Lim} T x t_{\text {r.e. }}[K] \subset \operatorname{Dis} Q_{K \text {-r.e. }}$.

Proof. First, we prove $\operatorname{Lim} T x t_{\text {r.e. }}[K] \subseteq \operatorname{Dis} Q_{K \text {-r.e. }}$ For that purpose, suppose

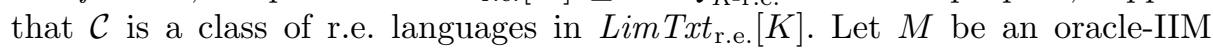
identifying $\mathcal{C}$ in the limit with respect to $\left(W_{i}\right)_{i \in \mathbb{N}}$, using a $K$-oracle.

Suppose $L \in \mathcal{C}$ is the target language. Let $\left(V_{i}\right)_{i \in \mathbb{N}}$ be a uniformly $K$-r.e. family, in which grammars for all queries as posed in the instructions below can be computed (* such a family exists *). A Dis Q-learner $M^{\prime}$ for $L$ with respect to $\left(V_{i}\right)_{i \in \mathbb{N}}$ is defined to act on the following instructions, starting in stage 0 . Stage $n$ reads as follows:

\footnotetext{
${ }^{5} \mathcal{C}_{R} \in \operatorname{Lim} T x t_{\text {r.e. }}[K]$ also follows from a result in [1], which proves that the access to an oracle for $K$ permits to learn the class of all recursive functions in the limit, as defined in [9]. This finally yields $L i m T x t_{\text {r.e. }}[K]$-learnability of $\mathcal{C}_{R}$.
} 
- Ask disjointness queries for $\left\{w_{0}\right\}, \ldots,\left\{w_{n}\right\}$. Let $L_{[n]}$ be the set of words $w_{x}, x \leq n$, for which the corresponding query is answered with 'no'.

(* Note that $L_{[n]}=L \cap\left\{w_{x} \mid x \leq n\right\} .{ }^{*}$ )

- Let $\left(\sigma_{x}^{n}\right)_{x \in \mathbb{N}}$ be an effective enumeration of all finite text segments for $L_{[n]}$. For all $x, y \leq n$ compute $M\left(\sigma_{x}^{y}\right)$ (* note that for these computations a $K$-oracle must be simulated $\left.{ }^{*}\right)$ as follows: whenever $M$ wants to access a $K$-oracle in order to determine whether $k \in K$ for some $k \in \mathbb{N}$, then pose a disjointness query for the language

$V_{k}^{\prime}= \begin{cases}\Sigma^{*}, & \text { if } k \in K, \\ \emptyset, & \text { otherwise }\end{cases}$

If the answer is 'yes', then transmit the answer 'no' to $M$ and vice versa.

- For each $x, y \leq n$, pose a disjointness query for the $K$-r.e. language $\overline{W_{M\left(\sigma_{x}^{y}\right)}}$. Let $\operatorname{Cand}_{n}=\left\{\sigma_{x}^{y} \mid x, y \leq n\right.$ and $\left.\overline{W_{M\left(\sigma_{x}^{y}\right)}} \cap L=\emptyset\right\}$ be the set of those segments, for which the query has been answered with 'yes'.

(* Note that $\operatorname{Cand}_{n}=\left\{\sigma_{x}^{y} \mid x, y \leq n\right.$ and $\left.\left.L \subseteq W_{M\left(\sigma_{x}^{y}\right)}\right)\right\} .{ }^{*}$ )

- For all $\sigma \in \mathrm{Cand}_{n}$, pose a disjointness query for the $K$-r.e. language

$V_{\sigma}^{\prime}= \begin{cases}\Sigma^{*}, & \text { if, given access to a } K \text {-oracle as requested, } \\ & M\left(\sigma \sigma^{\prime}\right) \neq M(\sigma) \text { for some text segment } \sigma^{\prime} \text { of } W_{M(\sigma)}, \\ \emptyset, & \text { otherwise }\end{cases}$

(* $V_{\sigma}^{\prime} \cap L=\emptyset$ iff $\sigma$ is a LimTxt-stabilizing sequence for $M$ and $\left.W_{M(\sigma)} .{ }^{*}\right)$

If all these queries are answered 'no', then go to stage $n+1$. Else, if $\sigma \in$ Cand $_{n}$ is minimal with $V_{\sigma}^{\prime} \cap L=\emptyset$, then hypothesize a $j$ with $V_{j}=W_{M(\sigma)}$ and stop.

$M^{\prime}$ identifies $L$ with disjointness queries in $\left(V_{i}\right)_{i \in \mathbb{N}}$; the proof is omitted. So $\mathcal{C} \in D i s Q_{K \text {-r.e. }}$ and $\operatorname{LimTxt}_{\text {r.e. }}[K] \subseteq \operatorname{Dis} Q_{K \text {-r.e. }}$.

$\operatorname{Lim} T x t_{\text {r.e. }}[K] \neq D i s Q_{K \text {-r.e. }}$ can be verified as follows:

We say that an oracle-IIM $M$ is nice, if for all oracles $A$ and all languages $L$, [if $M^{A}$ has a stabilizing sequence on $L$, then every text for $L$ starts with a stabilizing sequence for $M^{A}$ on $L$ ]. Note that from any oracle-IIM $M$, one can effectively find an oracle-IIM $M^{\prime}$ such that $M^{\prime}$ is nice, and for all $A, \operatorname{Lim}_{\text {Txt }}$ r.e. $[A]$-identifies at least as much as $M$ (a construction in [8] can be seen to easily relativize).

Thus, let $M_{0}, M_{1}, \ldots$ be a recursive sequence of nice oracle-IIMs, such that

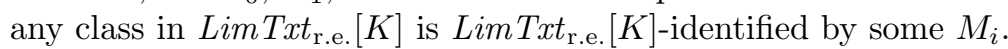

Let $X_{i}=\left\{a^{i} b^{x} \mid x \in \mathbb{N}\right\}$. Let $t^{i}$ be the canonical text $a^{i} b^{0}, a^{i} b^{1}, a^{i} b^{2}, \ldots$ for $X_{i}$. Let $X_{i}^{n}=$ content $\left(t_{n}^{i}\right)=\left\{a^{i} b^{x} \mid x \leq n\right\}$.

Define $L_{i}$ as follows. If there is no stabilizing sequence for $M_{i}^{K}$ on $X_{i}$, then let $L_{i}=X_{i}$. Else, let $t_{n}^{i}$ be a stabilizing sequence for $M_{i}^{K}$ on $X_{i}$ (where $n$ is the least non-zero number such that $t_{n}^{i}$ is a stabilizing sequence for $M_{i}^{K}$ on $X_{i}$ ). Then if $W_{M_{i}^{K}\left(t_{n}^{i}\right)} \supset X_{i}^{n}$, then let $L_{i}=X_{i}^{n}$; else let $L_{i}=X_{i}^{n+1}$.

Let $\mathcal{C}=\left\{L_{i} \mid i \in \mathbb{N}\right\}$. (* Note that $\mathcal{C}$ consists only of recursive languages. ${ }^{*}$ )

Note that $M_{i}^{K}$ does not $\operatorname{Lim} T x t_{\text {r.e. }}[K]$-identify $L_{i}$. Thus $\mathcal{C} \notin \operatorname{Lim} T x t_{\text {r.e. }}[K]$.

We now show how to get a $K$-r.e. grammar for $L_{i}$ from $i$. This is clearly enough to verify $\mathcal{C} \in D i s Q_{K \text {-r.e. }}$ (as $i$ can be obtained by asking disjointness queries for $L_{0}, L_{1}, \ldots$, until the unique $i$ to cause a 'no'-reply is found). 
Now $a^{i} b^{n} \in L_{i}$ iff: (i) $n=0$ or

(ii) $\forall y \leq n\left[t_{y}^{i}\right.$ is not a stabilizing sequence for $M_{i}^{K}$ on $\left.X_{i}\right]$ or

(iii) $\forall y<n\left[\left[t_{y}^{i}\right.\right.$ is not a stabilizing sequence for $M_{i}^{K}$ on $\left.X_{i}\right]$ and $\left.W_{M_{i}^{K}\left(t_{n}^{i}\right)} \not \supset X_{i}^{n}\right]$.

This is a $K$-r.e. predicate, hence one can obtain a $K$-r.e. grammar for $L_{i}$.

Theorem 9. $\operatorname{Dis} Q_{K \text {-r.e. }} \subset \operatorname{Sup} Q_{K \text {-r.e. }}$.

Proof. First we show $D i s Q_{K \text {-r.e. }} \subseteq \operatorname{Sup} Q_{K \text {-r.e. }}$. Let $\mathcal{C}$ be a class of r.e. languages $D i s Q$-learnable by some $M$ in a uniformly $K$-r.e. hypothesis space $\left(V_{i}\right)_{i \in \mathbb{N}}$. Let $\left(V_{i}^{\prime}\right)_{i \in \mathbb{N}}$ be a uniformly $K$-r.e. family, in which grammars for all superset queries needed below can be computed (* such a family exists *). For a target language $L$, an IIM $M^{\prime}$ is defined to execute stage 0 .

Stage $n$ : Simulate $M$. If $M$ poses a disjointness query for $V_{j}$, determine the set $\operatorname{Cand}_{n}$ of all $w \in\left\{w_{0}, \ldots, w_{n}\right\}$, for which a superset query concerning

$V_{w}^{\prime}= \begin{cases}\Sigma^{*}, & \text { if } w \in V_{j}, \\ \emptyset, & \text { if } w \notin V_{j},\end{cases}$

is answered with 'yes'. $\left({ }^{*} \operatorname{Cand}_{n}=\left\{w_{0}, \ldots, w_{n}\right\} \cap V_{j} .{ }^{*}\right)$

Then pose a superset query for all languages $\Sigma^{*} \backslash\{w\}$ with $w \in \operatorname{Cand}_{n}$. If all the answers are 'yes' (* $\left.\operatorname{Cand}_{n} \cap L=\emptyset *\right)$, then transmit the answer 'yes' to $M$, else transmit the answer 'no' to $M$. (* 'no'-answers are always correct. *)

If $M$ has not returned a hypothesis within $n$ steps, then go to stage $n+1$, else, if $M$ has guessed the language $V_{i}$, pose a superset query representing $V_{i}$ in $\left(V_{s}^{\prime}\right)_{s \in \mathbb{N}}$. If the answer is 'no', then go to stage $n+1$. If the answer is 'yes', then let $J$ be the set of indices of queries of $M$, which have been answered with 'yes'. For all $j \in J$ pose a superset query for

$V^{\prime}= \begin{cases}\Sigma^{*}, & \text { if } V_{j} \cap V_{i} \neq \emptyset, \\ \emptyset, & \text { if } V_{j} \cap V_{i}=\emptyset .\end{cases}$

If all these queries are answered 'no', then return a grammar for $V_{i}$ in $\left(V_{k}^{\prime}\right)_{k \in \mathbb{N}}$ (* because all queries are then answered correctly for the language $V_{i} \supseteq L$ and for $L$ - so the hypothesis $V_{i}$ of $M$ must be correct for $\left.L^{*}\right)$. Else go to stage $n+1$.

It is not hard to show that $M^{\prime}$ learns $L$ respecting $\left(V_{i}^{\prime}\right)_{i \in \mathbb{N}}$. So $\mathcal{C} \in \operatorname{Sup} Q_{K \text {-r.e. }}$. Second, a class in $\operatorname{Sup} Q_{K \text {-r.e. }} \backslash D i s Q_{K \text {-r.e. }}$ can be defined as follows.

Let $A$ be a $\Pi_{3}$-complete set. Let $L_{i}=\left\{a^{i} b^{j+1} a^{x+1} \mid j, x \in \mathbb{N}\right\}$ and $L_{i}^{s}=$ $\left\{a^{i} b^{j+1} a^{x+1} \mid j \in \mathbb{N}, x \leq s\right\}$ for all $i, s \in \mathbb{N}$. Finally, let $\mathcal{C}=\left\{L_{i} \mid i \in A\right\} \cup\left\{L_{i}^{s} \mid\right.$ $i \notin A, s \in \mathbb{N}\}$. (* Note that $\mathcal{C}$ consists only of recursive languages. ${ }^{*}$ )

We first show $\mathcal{C} \notin D i s Q_{K-\text {-r.e. }}$. Suppose by way of contradiction that $M$ witnesses $\mathcal{C} \in D i s Q_{K \text {-r.e. }}$ in some uniformly $K$-r.e. family $\left(V_{i}\right)_{i \in \mathbb{N}}$. We establish a contradiction by concluding $A \in \Sigma_{3}$, though $A$ is $\Pi_{3}$-complete. For that purpose, fix recursive sets $Q, R$ with $i \in A$ iff $\forall x \exists y \forall z[Q(i, x, y, z)] ; w \in V_{i}$ iff $\exists y \forall z[R(i, w, y, z)]$. Define a $\Sigma_{3}$-procedure $P$ on input $i \in \mathbb{N}$ to begin in stage 0 .

Stage $n$ : a) Test whether or not $\exists y \forall z[Q(i, n, y, z)]$ is true. If not, then stop with the output ' $i \notin A$ '. Else go to b). 
b) Simulate $M$ for $n+1$ steps. Thereby, whenever $M$ poses a disjointness query $k$, transmit the answer 'yes' to $M$ in case $\exists j, x \exists y \forall z\left[R\left(k, a^{i} b^{j+1} a^{x+1}, y, z\right)\right]$ is not true (*i.e., if $L_{i} \cap V_{k}=\emptyset *$ ); the answer 'no', otherwise. In case $M$ does not return any hypothesis within $n+1$ steps of computation, go to stage $n+1$. Else stop with the output ' $i \in A$ '. (* If $i \notin A$, then there would be some $s$, such that, in the scenario above, all answers transmitted to $M$ would be correct for the languages $L_{i}^{s}, L_{i+1}^{s}, L_{i+2}^{s}, \ldots$, which would all belong to $\mathcal{C}$. Since $M$ returns a hypothesis, $M$ would fail for infinitely many languages in $\mathcal{C}$-a contradiction. ${ }^{*}$ )

$P$ decides $A$ in $\Sigma_{3}$. This contradiction implies that $\mathcal{C} \notin D i s Q_{K \text {-r.e. }}$.

We now show that $\mathcal{C} \in \operatorname{Sup} Q_{K \text {-r.e. }}$.

Let $q_{x}^{i}(t)=\min (\{t\} \cup\{r \leq t \mid \forall z \leq t[Q(i, x, r, z)]\})$. Note that $q_{x}^{i}(t)$ is recursive, and $\lim _{t \rightarrow \infty} q_{x}^{i}(t)$ exists iff $\exists y \forall z[Q(i, x, y, z)]$ is true. Let

$f_{i}(u, t)= \begin{cases}1, & \text { if } \forall x \leq u\left[q_{x}^{i}(t)=q_{x}^{i}(t+1)\right], \\ 0, & \text { otherwise. }\end{cases}$

It is easy to verify that $X_{i}=\left\{w_{u} \mid \lim _{t \rightarrow \infty} f_{i}(u, t)=1\right\}$, is finite if $i \notin A$, and equal to $\Sigma^{*}$, if $i \in A$. Moreover, $f_{i}$ is a $K$-r.e. function for $X_{i}$. Thus, a $K$-r.e. grammar for $X_{i}$ can be obtained effectively from $i$.

Now define $M$ as follows. For a target language $L$, query $\Sigma^{*} \backslash\left\{a^{i} b^{j+1} a^{x+1}\right\}$, for various values of $i, j, x$, until $i, j, x$ are found such that $\Sigma^{*} \backslash\left\{a^{i} b^{j+1} a^{x+1}\right\} \nsupseteq L$. By definition of $\mathcal{C}$, this implies that $L$ is of the form $L_{i}$ or $L_{i}^{s}$ for some $s \in \mathbb{N}$. Now, pose a superset query for $X_{i}$. Note that if $i \in A$, then $X_{i}=\Sigma^{*} \supseteq L$, and if $i \notin A$, then $X_{i} \nsupseteq L$ (as $X_{i}$ would then be finite, while $L$ is infinite). Thus $M$ can determine whether or not $i \in A$. If $i \in A$, then $M$ outputs a grammar for $L_{i}$. If not, then $M$ searches for the minimal $s \in \mathbb{N}$ such that $L_{i}^{s} \supseteq L$. Now $L=L_{i}^{s}$, and $M$ can output a grammar for $L_{i}^{s}$. Thus $\mathcal{C} \in S u p Q_{K \text {-r.e. }} \backslash \operatorname{Dis} Q_{K \text {-r.e. }}$

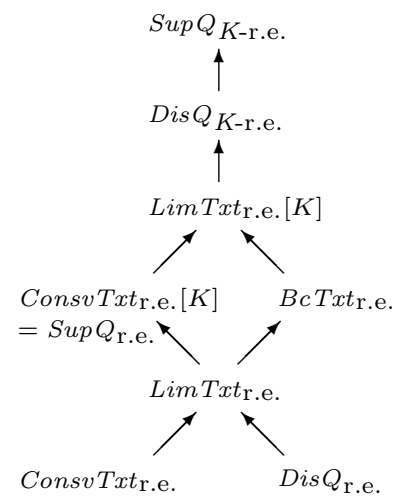

Figure 2. This graph illustrates the relations between different inference types studied above. Arrows indicate proper inclusions of inference types. Two inference types which are not connected by a path of arrows are incomparable.

\section{Discussion}

Above we have seen that many of the equivalences of Gold-style and query inference types in the context of learning indexable classes no longer hold, if 
arbitrary target classes of r.e. languages are considered. Nevertheless, these two approaches of learning reveal strong relations, expressed in an inclusion hierarchy of inference types. Altogether, this shows that in many cases all learners of the one kind of inference types can be transformed into learners of the other kind without loss of learning power, though in general not vice versa. Interestingly, our proofs for the inclusions are constructive, i.e. the transformations of learners can be done uniformly and indicate the essential reasons for the strong relations.

Another outcome is that all our separation results are witnessed by classes of recursive languages: $\operatorname{Sup} Q_{\text {r.e. }} \backslash \operatorname{Lim} T x t_{\text {r.e. }} \neq \emptyset, B c T x t_{\text {r.e. }} \backslash \operatorname{Sup} Q_{\text {r.e. }} \neq \emptyset$, and $\operatorname{Lim} T x t_{\text {r.e. }}[K] \backslash S u p Q_{\text {r.e. }} \neq \emptyset$ are obtained in $[14,15]$ using indexable classes of recursive languages; the other separations displayed in Figure 2 have been verified with non-indexable classes of recursive languages. For the latter, note that all classes used in our proofs above consist only of recursive languages. Of course these proofs would not have worked with indexable classes of recursive languages, since the corresponding separations do not hold for indexable classes, see Figure 1. So the equalities are not due to the recursiveness of the target languages alone. The fact that the target classes are indexable is crucial. This raises the question whether the new inequalities obtained above hold for uniformly r.e. classes. As it turns out, at least one of them does not, while some of them do.

When restricting the focus to learning indexable classes, [14] has shown that the capabilities of $D i s Q_{K \text {-r.e. }}$-learners and $\operatorname{Lim} T x t_{\text {r.e. }}[K]$-learners are equal, which does not hold for general classes of recursive languages, as witnessed in the proof of Thm. 8. Interestingly, the enumerability of the target class is the crucial reason for the equality result in [14], as the following theorem illustrates.

Theorem 10. Let $\mathcal{C}$ be uniformly r.e. Then $\mathcal{C} \in \operatorname{Dis} Q_{K \text {-r.e. }}$ iff $\mathcal{C} \in \operatorname{LimTxt}_{\text {r.e. }}[K]$.

Proof. $\operatorname{Lim} T x t_{\text {r.e. }}[K] \subseteq D i s Q_{K \text {-r.e. }}$ by Thm. 8. So suppose $\mathcal{C} \in D i s Q_{K \text {-r.e. }}$ is uniformly r.e. Let $f$ be a recursive function such that $\mathcal{C}=\left\{W_{f(i)} \mid i \in \mathbb{N}\right\}$. Let $M$ be a $D i s Q_{K \text {-r.e. }}$-learner for $\mathcal{C}$ in a $K$-r.e. hypothesis space $\left(V_{i}\right)_{i \in \mathbb{N}}$. Let $g$ be a recursive function such that $w \in V_{i}$ iff $\lim _{t \rightarrow \infty} g(i, w, t)=1$.

The idea is to construct a $\operatorname{Lim} T x t_{\text {r.e. }}[K]$-learner $M^{\prime}$ for $\mathcal{C}$ by simulating $M$. Given a text segment $t_{n}, M^{\prime}$ searches for a language in $\mathcal{C}$, which is consistent with $t_{n}$ and for which the behaviour of the known learner $M$ seems reasonable, at least when taking $t_{n}$ into consideration. The length of the given text segment serves as a bound for $M^{\prime}$ when trying to analyse whether the behaviour of $M$ is reasonable. Define $M^{\prime}\left(t_{n}\right)$ (using an oracle for $K$ ) as follows:

- If there exists a $j \leq n$ such that the following three conditions are satisfied: 1. $\operatorname{content}\left(t_{n}\right) \subseteq W_{f(j)}$.

2. $M$ outputs a hypothesis if the questions $k$ of $M$ are answered as follows: - 'no', if there exists a $w \in$ content $\left(t_{n}\right)$ with $g\left(k, w, n^{\prime}\right)=1$ for all $n^{\prime} \geq n$. - 'yes', otherwise.

3. For any query $k$ made by $M$ in the simulation in 2 above: if there exists a $w \in W_{f(j), n}$ with $g\left(k, w, n^{\prime}\right)=1$ for all $n^{\prime} \geq n$, then there also exists a $w \in \operatorname{content}\left(t_{n}\right)$ with $g\left(k, w, n^{\prime}\right)=1$ for all $n^{\prime} \geq n$. (* i.e., the seeming 'yes'-answers for content $\left(t_{n}\right)$ as a target language also seem to be 'yes'-answers for $W_{f(j)}$ as a target language $\left.*\right)$. 
then output $f(j)$ for the least such $j$. Else output 0 .

Note that these simulations can be done using an oracle for $K$. We now claim

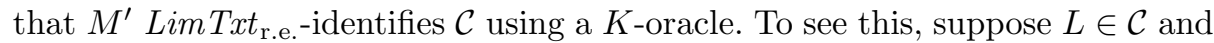
$t$ is a text for $L$. Let $j$ be minimal with $W_{f(j)}=L$. Fix $n$ large enough such that:

A. If all the questions of $M$ are answered correctly in the simulation made by $M^{\prime}\left(t_{n}\right)$, then $M$ outputs a hypothesis.

B. $j \leq n$.

C. Let $Q$ denote the set of questions asked as in A above. Then, for all $k \in Q$, if $V_{k} \cap L \neq \emptyset$, then for some $w \in \operatorname{content}\left(t_{n}\right)$, for all $n^{\prime} \geq n, g\left(k, w, n^{\prime}\right)=1$ (* i.e., answers can be given correctly based on $t_{n} *$ ).

D. For all $j^{\prime}<j$, if $W_{f\left(j^{\prime}\right)} \nsupseteq L$, then $W_{f\left(j^{\prime}\right)} \nsupseteq$ content $\left(t_{n}\right)$.

E. For all $j^{\prime}<j$, if $W_{f\left(j^{\prime}\right)} \supset L$, then for some $k \in Q$ with $V_{k} \cap L=\emptyset$ and $x \in W_{f\left(j^{\prime}\right), n}$, for all $n^{\prime} \geq n, g\left(k, x, n^{\prime}\right)=1$.

Such an $n$ exists (for (A-D), clearly; for (E), if for $j^{\prime}<j$ no such $n$ existed, then $t_{n}$ could be extended to a text segment for $W_{f(j)}$ and $W_{f\left(j^{\prime}\right)}$, with answers as in (A) being correct for both $W_{f(j)}$ and $W_{f\left(j^{\prime}\right)}$. So $M$ would fail to $D i s Q_{\text {r.e. }}{ }^{-}$ learn at least one of them). Now, $M^{\prime}\left(t_{n^{\prime}}\right)=f(j)$ follows for all $n^{\prime} \geq n$.

For the other separations, except for $\operatorname{Sup} Q_{K \text {-r.e. }} \backslash D i s Q_{K \text {-r.e. }} \neq \emptyset$, we will now prove that even enumerability of the target class is not sufficient for achieving the equality results from $[14,15]$. Whether or not a similar result holds for the separation of $\operatorname{Sup} Q_{K \text {-r.e. }}$ and $\operatorname{Dis} Q_{K \text {-r.e. }}$, remains an open question.

Theorem 11. 1. There exists a uniformly r.e. class in Lim Txt $t_{\text {r.e. }} \backslash D i s Q_{\text {r.e. }}$

2. There exists a uniformly r.e. class in $S u p Q_{\text {r.e. }} \backslash B c T x t_{\text {r.e. }}$.

3. There exists a uniformly r.e. class in LimTxt $t_{\text {r.e. }}[K] \backslash B c T x t_{\text {r.e. }}$.

Proof. ad 1. This is already witnessed by the proof of Thm. 2, where a uniformly r.e. class of recursive languages is used for the separation.

ad 2 and 3. The idea is to use a class comprising the class $\mathcal{C}_{R}$ used in the proof of Thm. 6. For that purpose, choose a uniformly r.e. family $\left(L_{i}\right)_{i \in \mathbb{N}}$ of recursive languages satisfying the following demands:

- for all $i \in \mathbb{N}, L_{i}$ is either finite or $L_{i} \in \mathcal{C}_{R}$,

- for all $L \in \mathcal{C}_{R}$ there is some $i \in \mathbb{N}$ such that $L=L_{i}$,

$-L_{0}=\emptyset$,

- for all $i, j \in \mathbb{N}$ with $L_{i} \neq \emptyset$, if $L_{i} \subset L_{j}$, then $i<j$.

Such a family can be constructed with standard methods. Now, if $\mathcal{C}=\left\{L_{i} \mid i \in\right.$ $\mathbb{N}\}$, then $\mathcal{C}$ obviously comprises $\mathcal{C}_{R}$ and thus $\mathcal{C} \notin B c T x t_{\text {r.e. }}$ In contrast to that, $\mathcal{C} \in S u p Q_{\text {r.e }}$ : for identifying some $L \in \mathcal{C}$ a query learner can find the least $i$ such that $L_{i} \supseteq L$. The properties of $\mathcal{C}$ then imply $L_{i}=L$.

Thus, $\mathcal{C} \in S u p Q_{\text {r.e. }}$ and, by Thm. 5 , also $\mathcal{C} \in \operatorname{Lim}_{\operatorname{Txt}}$ r.e. $[K]$.

Thus we have seen that in several cases the equivalence results for indexable classes from previous work are diminished to strict inclusions, regardless of whether or not the target class (i) consists of recursive languages only, or (ii) 
is enumerable (the latter with one exception, see Thm. 10). This shows that indexable target classes yield a specific situation for Gold-style and query learning. Strong relationships between the two models are already witnessed in the general case of learning arbitrary classes of r.e. languages, but only a restriction to even indexable target classes further intensifies these relationships.

\section{Acknowledgement}

Many thanks are due to the anonymous referees for their helpful comments, especially, for a simplified proof of Theorem 11-(2).

\section{References}

1. L. Adleman, M. Blum. Inductive inference and unsolvability. J. Symbolic Logic, 56:891-900, 1991.

2. D. Angluin. Inductive inference of formal languages from positive data. Inform. Control, 45:117-135, 1980.

3. D. Angluin. Queries and concept learning. Machine Learning, 2:319-342, 1988.

4. D. Angluin. Queries revisited. Theoret. Comput. Sci., 313:175-194, 2004.

5. J. Barzdins. Two theorems on the limiting synthesis of functions. Theory of Algorithms and Programs, Latvian State University, Riga 210:82-88, 1974.

6. J. Case, C. Lynes. Machine inductive inference and language identification. In: Proc. ICALP 1982, LNCS 140, pp. 107-115, Springer, 1982.

7. D. De Jongh, M. Kanazawa. Angluin's theorem for indexed families of r.e. sets and applications. In: Proc. COLT 1996, pp. 193-204, ACM Press, 1996.

8. M. Fulk. Prudence and other conditions for formal language learning. Inform. Comput., 85:1-11, 1990.

9. E. M. Gold. Language identification in the limit. Inform. Control, 10:447-474, 1967.

10. J. E. Hopcroft, J. D. Ullman. Introduction to Automata Theory, Languages, and Computation. Addison-Wesley Publishing Company, 1979.

11. S. Jain, E. Kinber. Learning languages from positive data and negative counterexamples. In: Proc. ALT 2004, LNAI 3244, pp. 54-68, Springer, 2004.

12. S. Jain, E. Kinber. Learning languages from positive data and a finite number of queries. In: Proc. FSTESTCS 2004, LNAI 3328, pp. 360-371, Springer, 2004.

13. S. Lange, T. Zeugmann. Language learning in dependence on the space of hypotheses. In: Proc. COLT 1993, pp. 127-136, ACM Press, 1993.

14. S. Lange, S. Zilles. Replacing limit learners with equally powerful one-shot query learners. In: Proc. COLT 2004, LNAI 3120, pp. 129-143, Springer, 2004.

15. S. Lange, S. Zilles. Comparison of query learning and Gold-style learning in dependence of the hypothesis space. In: Proc. ALT 2004, LNAI 3244, pp. 99-113, Springer, 2004.

16. S. Lange, S. Zilles. Relations between Gold-style learning and query learning. Submitted to Inform. Comput.

17. H. Rogers, Jr. Theory of Recursive Functions and Effective Computability, MIT Press, 1987.

18. F. Stephan. Degrees of Computing and Learning. Habilitationsschrift, RuprechtKarls-Univ., Heidelberg, 1999.

19. T. Zeugmann, S. Lange. A guided tour across the boundaries of learning recursive languages. In: Algorithmic Learning for Knowledge-Based Systems, LNAI 961, pp. 190-258, Springer, 1995. 